\title{
An Echo State Network Approach to Structural Health Monitoring
}

\author{
A. J. Wootton, C. R. Day, P. W. Haycock \\ Keele University \\ Staffordshire \\ United Kingdom \\ $\{a . j . w o o t t o n$, c.r.day,p.w.haycock\}@keele.ac.uk
}

\begin{abstract}
Echo State Networks (ESNs) have been applied to time-series data arising from a structural health monitoring multi-sensor array placed onto a test footbridge which has been subjected to a number of potentially damaging interventions over a three year period. The time-series data, sampled approximately every five minutes from ten temperature sensors, have been used as inputs and the ESNs were tasked with predicting the expected output signal from eight tilt sensors that were also placed on the footbridge. The networks were trained using temperature and tilt sensor data up to the first intervention and subsequent discrepancies in the ESNs' prediction accuracy allowed inferences to be made about when further interventions occurred and also the level of damage caused. Comparing the error in signals with the location of each of the tilt sensors allowed damaged regions to be determined.
\end{abstract}

Keywords-Structural Health Monitoring; Echo State Networks; Reservoir Computing Applications; Wireless Sensor Networks

\section{INTRODUCTION}

Intelligent analysis of data collected from reinforced concrete structures using non-destructive techniques is becoming increasingly important given the increased availability and use of non-invasive structural health monitoring (SHM) sensor networks that can monitor our built civil engineering infrastructure in real time. Such analyses have the potential to provide structural engineers with a more accurate picture of the dynamics of structures and an indication of the presence of defects within a structure: a task which hitherto has been difficult and costly. Early detection of defects within a structure not only helps to increase the serviceable life of a structure and prevent structural failure, but also reduces repair costs due to a reduction in the severity of any defects.

In this study, a type of Recurrent Neural Network (RNN), the Echo State Network (ESN), was applied to a very large, multi-dimensional, longitudinal, time-series dataset composed of sensor readings from a real-world civil engineering structure that was subjected to a number of deliberate interventions, many of which were likely to undermine the structure's integrity. This study built on previous work, where the present authors compared the

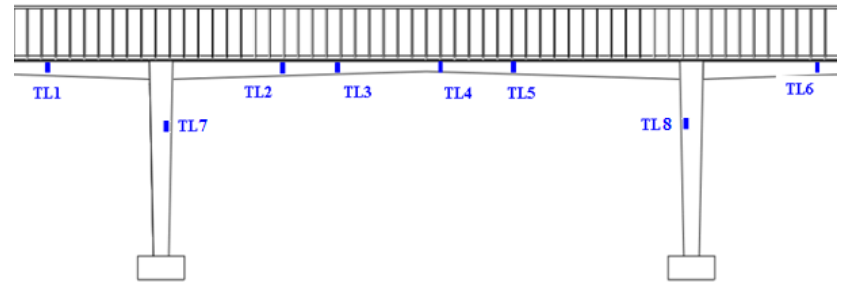

Figure 1: The locations of the eight tilt sensors, labelled TL1-8, on the bridge.

ESN approach to the commonly used NARMAX model [1].

\section{A. The NPL Footbridge}

The UK's National Physical Laboratory (NPL) footbridge project was set up as a means of developing new SHM sensor technologies and the methods for processing large time-series datasets that arise from wireless sensor networks. The project was centred on a concrete footbridge that was built in the 1960s and underwent normal use for nearly 50 years prior to the beginning of the project. In 2009 it was taken out of use and was embedded with a plurality of sensors, which took data readings at regular five minute intervals over a three year period. The work we present here is concerned with the data produced by ten temperature sensors and eight tilt sensors, delivering 365376 sensor readings collected between January 2009 and May 2012. Fig. 1 shows the spatial arrangement of the tilt sensors on the bridge. Note that sensors 7 and 8 are attached to the two columns of the bridge and that it is a standalone structure, allowing weights to be suspended from either of the two cantilevers. This is due to the fact that for the duration of the study the bridge's sole purpose was for field testing. During the course of the study, the bridge was subjected to damage and repair cycles, detailed in full by Livina [2]. There have been other studies looking into the data provided by the project, but as of yet none has been able to accurately detect events and characterise any consequential long term damage to the bridge [2-5]. 


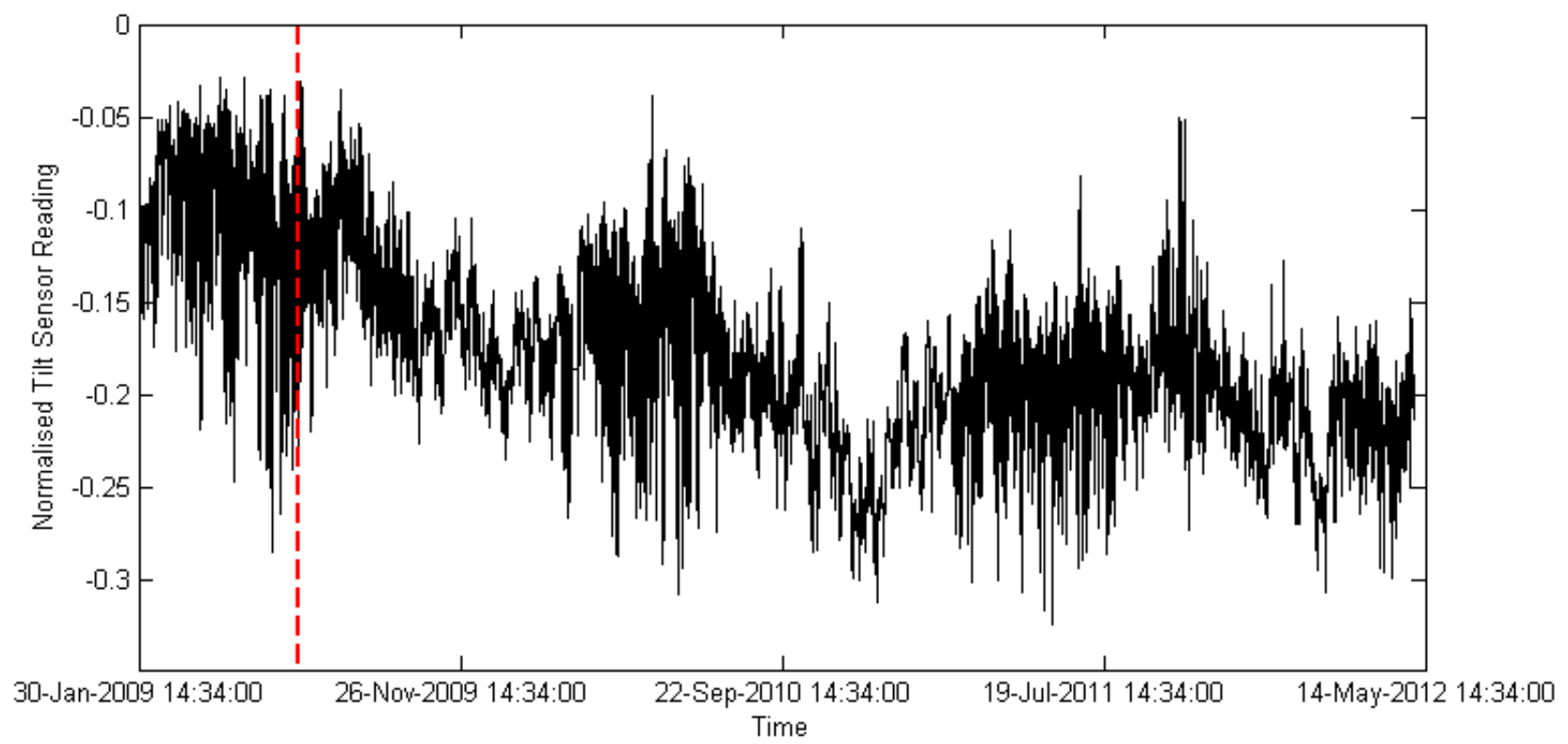

Figure 2: The data produced by tilt sensor 3, where the data to the left of the dashed vertical line were recorded prior to the first significant intervention and were hence used for training the network so that it replicated the ideal behaviour of the tilt sensors.

\section{B. Echo State Networks}

ESNs [6] offer a fast and efficient training procedure, making their application to real-world data very appealing and allowing them to overcome the problems usually associated with RNN training [7]. They have already been applied in domains as diverse as time-series prediction [8], robotics [912], speech recognition [13] and non-linear audio processing [14]. More recently, they have been used in domains such as electric load forecasting [15], sports activity classification [16] and shape recognition [17]. ESNs have also previously been used in a structural health monitoring context: for fault diagnosis in a water network [18] and defect detection in reinforced concrete [19]. In all of these studies, ESNs were

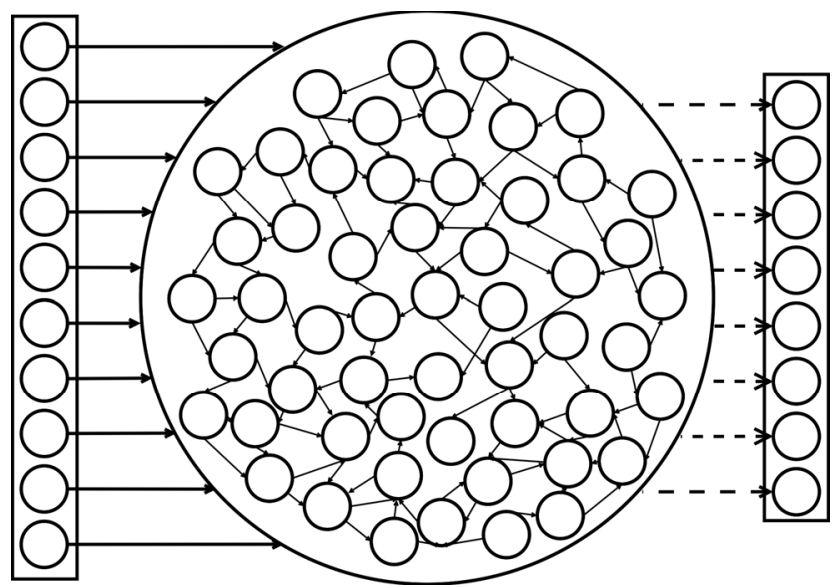

Figure 3: A schematic diagram of the ESN used here. Each solid arrow represents a connection generated randomly, while each dashed arrow represents a weight that was trained by ridge regression based on the real output of the corresponding tilt sensor. found to equal, or outperform, a number of other state-of-theart techniques.

An ESN architecture involves an input layer, a sparsely and randomly interconnected layer (the reservoir) and an output layer of artificial neurons, each of which is connected in the forward direction to its neighbouring layers. Once the reservoir neuron activations have been harvested after processing all of the data in the training dataset, only the output weights need to be adjusted to complete the training, using a simple linear regression technique, such as ridge regression [20].

Importantly, unlike most recurrent neural networks, it is only the connections between the reservoir neurons and the output units that are trained. All other weights, randomly generated at the start of the process, are kept constant. Proper tuning of a small number of network parameters (e.g. the spectral radius for reservoir neurons) gives rise to the 'echo state property' that allows the ESN to recall past inputs in the manner of a short term memory (STM). The STM effect decays asymptotically over each time step, with the speed at which this occurs depending on a few other parameters of the network. The 'echo state property' allows ESNs to capture temporal relationships between input and output data, which is the primary reason why they were chosen for this study. In this case, the temporal relationship between the temperature sensors and tilt sensors was under investigation.

The activation of the ESN reservoir units is evaluated in accordance with (1).

$$
\mathbf{x}(t)=f\left((1-\delta) \mathbf{x}(t-1)+\delta\left(\mathbf{W}_{r e s}^{\text {inp }} u(t)+\mathbf{W}_{\text {res }}^{\text {res }} \mathbf{x}(t-1)\right)\right)
$$

In (1), $\mathbf{W}_{\text {res }}^{\text {inp }}$ is the input to reservoir weight matrix, $\mathbf{x}(\mathrm{t})$ is the vector of the activations of the reservoir neurons at time $t$, 
which is the current time step, $\mathrm{t}-1$ is the previous time step, $\mathbf{W}_{\text {res }}^{\text {res }}$ is the reservoir weight matrix (drawn randomly from a $\mathrm{Z}$ distribution), $\mathbf{u}(\mathrm{t})$ is the vector of the input data at time $\mathrm{t}$ and $\delta$ is the leak rate, which determines the extent to which ESN reservoir neurons' activation decreases over a period of time. The activation function of the neurons is represented by $\mathrm{f}$ and in this case, a tanh activation function was used. The reservoir weight matrix is dependent on the desired spectral radius $\alpha$, as seen in (2).

$$
\mathbf{W}_{\text {res }}^{\text {res }}=\alpha \times \mathbf{W}_{\text {res }}^{\text {res }} /\left|\lambda_{\max }\right|
$$

In (2), $\lambda_{\max }$ is the maximum eigenvalue of $\mathbf{W}_{r e s}^{\text {res }}$, which represents the initial reservoir weights. The final outputs of the network for each output node are then calculated using (3).

$$
\mathbf{y}(t)=f^{\text {out }}\left(\mathbf{W}_{\text {out }}^{\text {res }}(\mathbf{x}(t))\right)
$$

In (3), $f^{\text {out }}$ is the activation function of the output unit, which is linear.

\section{EXPERIMENTAL SETUP}

In previous work, one data point per hour was sampled from the full dataset and then used to create a dataset that was split so that $70 \%$ was used for training and $30 \%$ for testing [1]. This approach worked well for detecting the most significant interventions, but it was not possible to see whether or the extent to which bridge had been damaged. This was due to the fact that a number of significant interventions had already taken place in the portion of data reserved for training. That meant that those ESNs were being trained to treat some of the intervention-affected behaviour of the tilt sensors as typical behaviour of the bridge.
Alternatively, the approach in the current work is to use only the data prior to the first significant intervention for training purposes and then to apply the trained ESNs to the remainder of the data. This approach means that the ESNs used were trained on the behaviour of the tilt sensors under only strictly normal conditions. Accordingly, the temporal extent of the data available for training was smaller than that used previously [1], so in the current work no sampling was performed and all of the available sample points were used instead. The data were normalised between -1 and +1 , a commonly used preprocessing technique that delivers improved RNN performance [21]. Fig. 2 shows the full set of data for just tilt sensor 3, with the training portion of the data being that which is to the left of the dashed vertical line. Initial investigations identified an ESN topology that seemed to deliver best performance using 10 input units, 500 reservoir units, 8 output units and a spectral radius of 0.9 . Since the weights of an ESN are random at network creation, 100 ESNs using this topology were simulated and the output for each tilt sensor averaged. This averaged output is the basis for the results presented below. The ESNs were simulated using Schrauwen, Verstraeten and d'Haene's Reservoir Computing Toolbox for MATLAB [22]. A schematic diagram of the topology used is given in Fig. 3.

The training regime outlined above meant that the ESNs would be trained to replicate the behaviour of the bridge in its initial state. Discrepancies between the ideal tilt sensor output produced by the ESN and the real tilt sensor output would thus be indicative of a change from this initial state and consequently, of damage to the bridge. Specifically, a significant divergence between the data recorded by each tilt sensor and the normal behaviour of the bridge as predicted by the ESN, taken as an increase in error of at least 0.01, was used to indicate that a significant event had occurred. Data recorded by NPL scientists meant that ground truth was available; the

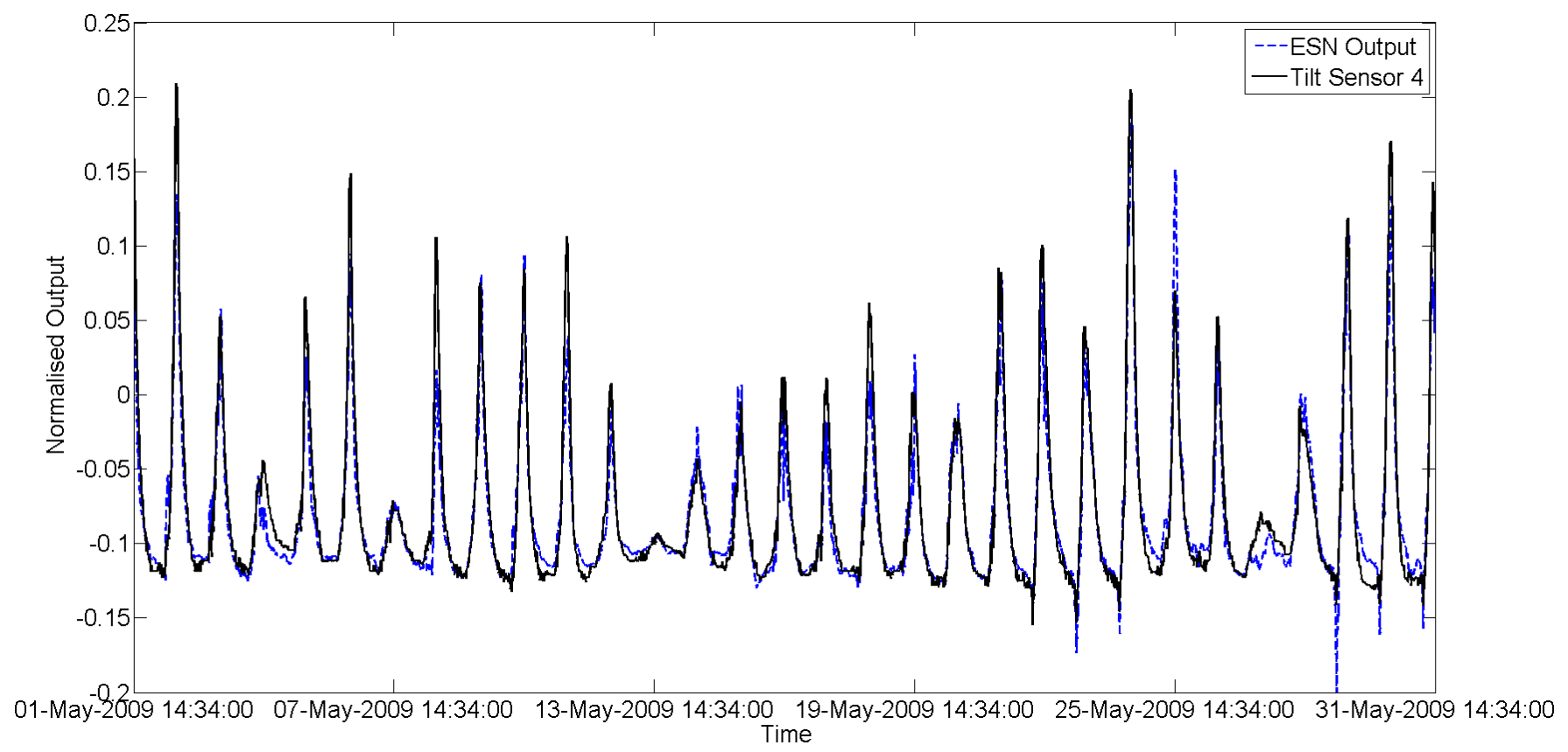

Figure 4: The output of tilt sensor 4 (solid line) compared with the ESN prediction for tilt sensor 4 (dashed line) over the month of May 2009. 
time and date of the events had been recorded, making it possible to localise the onset and cessation times of these events. In a 'real-world' scenario of course this information would not be available. However, the NPL scientists did not record the extent of any damage that might have been caused.

For each tilt sensor, the error between the data predicted by the ESN and the actual data at each point was calculated and then the modulus of a moving average of these values was calculated for a point $n$ in the time series according to (4):

$$
\begin{gathered}
x=\mid\left(P_{n-5000}+P_{n-4999}+\ldots+P_{n+4999} / 10000\right)-\left(A_{n-5000}+\right. \\
A_{n-4999}+\ldots+A_{n+4999} / 10000 \mid
\end{gathered}
$$

In (4), $x$ is the modulus of the final moving average value, $A$ is the actual data point from that tilt sensor and $P$ is the data point that was predicted by the ESN for that sensor. The 10000 point moving average covered a time period of approximately 34 days. This moving average error method was employed to help address the inherent noise in the dataset that could otherwise lead to useful information being obscured.

\section{RESULTS}

An example of the good correlation between real and predicted tilt sensor data in the training portion can be seen in Fig. 4, which shows the close match between the two sets of data for tilt sensor 4 over a period of a month in the spring of 2009. This is significant for two reasons. Firstly, it shows that the ESNs were able to accurately predict how the bridge should normally behave, strongly implying that any difference between the real and predicted values in the testing portion of the dataset would be due to a change in the state of the bridge, rather than a fault in the ESNs' prediction capability. Secondly, this demonstrates the same ability to follow the daily cycle of the tilt sensors as the support vector regression used by Kromanis and Kripakaran on the same data [4].

Using (4) to analyse the data produced by the ESNs resulted in two key damage events being identified, both involving the loading of water tanks from the cantilever containing sensor 1. Fig. 5 shows the error between tilt sensor 1 and the ESN prediction for tilt sensor 1 for the period $30^{\text {th }}$ January 2009 to $6^{\text {th }}$ March 2010, sampled at one data point per hour but without being smoothed according to (4). It was found that the greatest short term response to the loading of water tanks from the cantilever was seen in the unsmoothed error data from tilt sensor 1, as expected. It can be seen in Fig. 5 that the error for tilt sensor 1 sharply rises (to 1.30 in the first instance and 1.35 in the second instance) and falls (to 0.01 in the first instance and 0.05 in the second instance) as soon as the loading event starts and ends, respectively.

Fig. 6 shows a moving average of the error between the predicted data and the actual data for tilt sensors 2, 3 and 8 around two key points. Each solid vertical line represents an intervention involving the loading of water tanks onto one end of the bridge. A rise in the error level due to an event that is

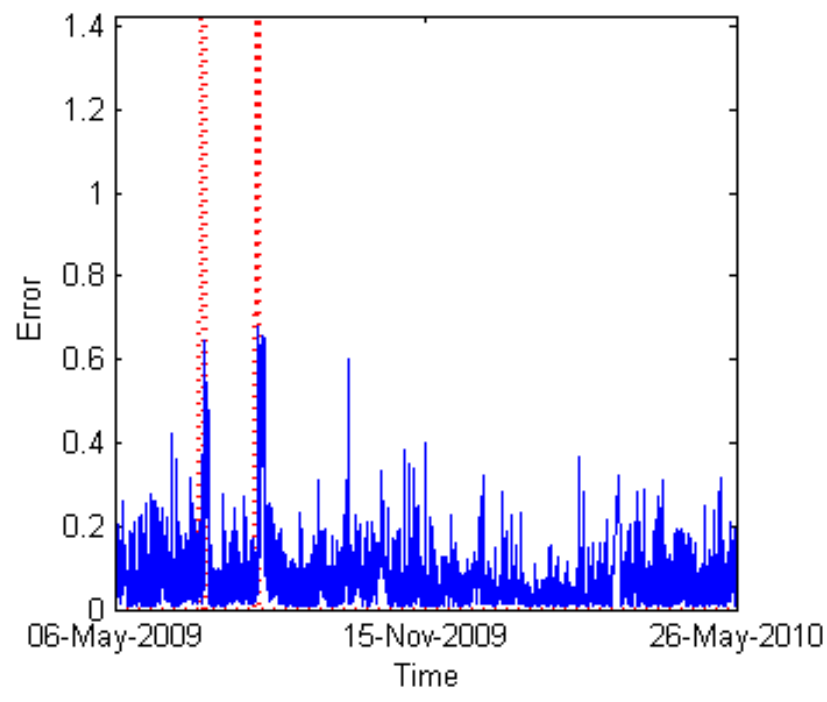

Figure 5: The error for tilt sensor 1. Note that here, the data has not been smoothed over 10000 points and is instead sampled at one data point per hour. The dashed vertical lines indicate two separate interventions, separated by a few days. Both of these involved the loading of a water barrel from the cantilever of the bridge.

then maintained over a long time period indicates that the event caused permanent damage, or at least a medium term change in the disposition of the bridge. A rise in the error level following the cessation of an event but which is followed by the error returning quickly to its prior level is indicative of the event affecting the bridge just for the duration of the event, but not causing any lasting damage. If there is no change in the error level due to an event then it is postulated that it did not affect the bridge in any significant way.

\section{DISCUSSION}

The first of the two key interventions found using this technique occurred in August 2009, when two water tanks were suspended from one end of the bridge and then filled with water and subsequently emptied. The data for the onset of this event is shown in Fig. 6A (vertical line) and it can be seen to have had a large effect on sensors 2, 3 and 8. For tilt sensor 2, the error rose from 0.01 to 0.16 , while for sensor 3 it rose from 0.00 to 0.08 and for sensor 8 from 0.01 to 0.03 .

The effect on sensors 2 and 3 was maintained not just immediately after the event onset, but over a sustained period of several months up to the point seen in Fig. 6A where the error stops increasing and plateaus. At the same time, the small increase in error for sensor 8, attached to the column, can be seen to return back to its original level, where a timescale for recovery of several months can be observed. From Fig. 5 it can also be seen that after the initial response of the bridge to the loading, which is largest for sensor 1, being on the cantilever, there is also a longer term response in this region that recovers at about the same time as the column. 
A
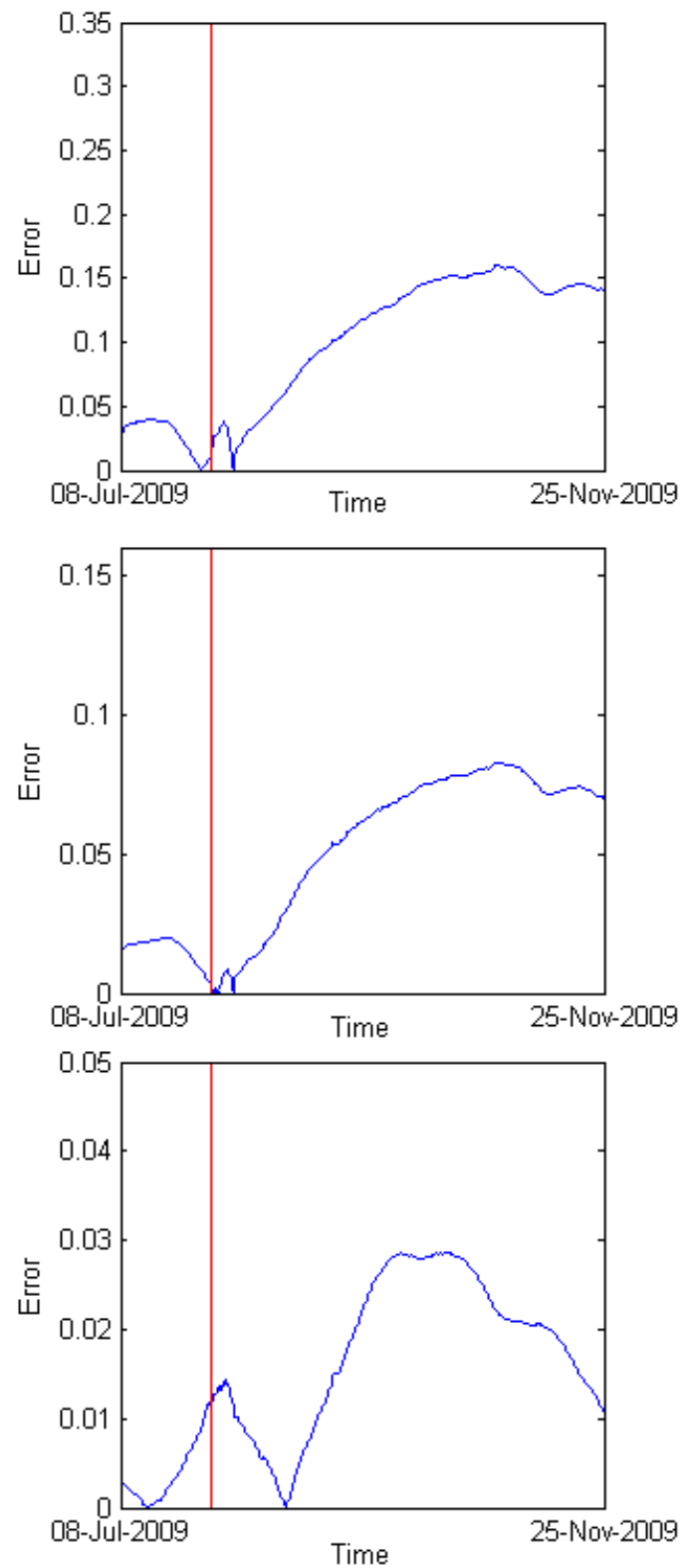

B
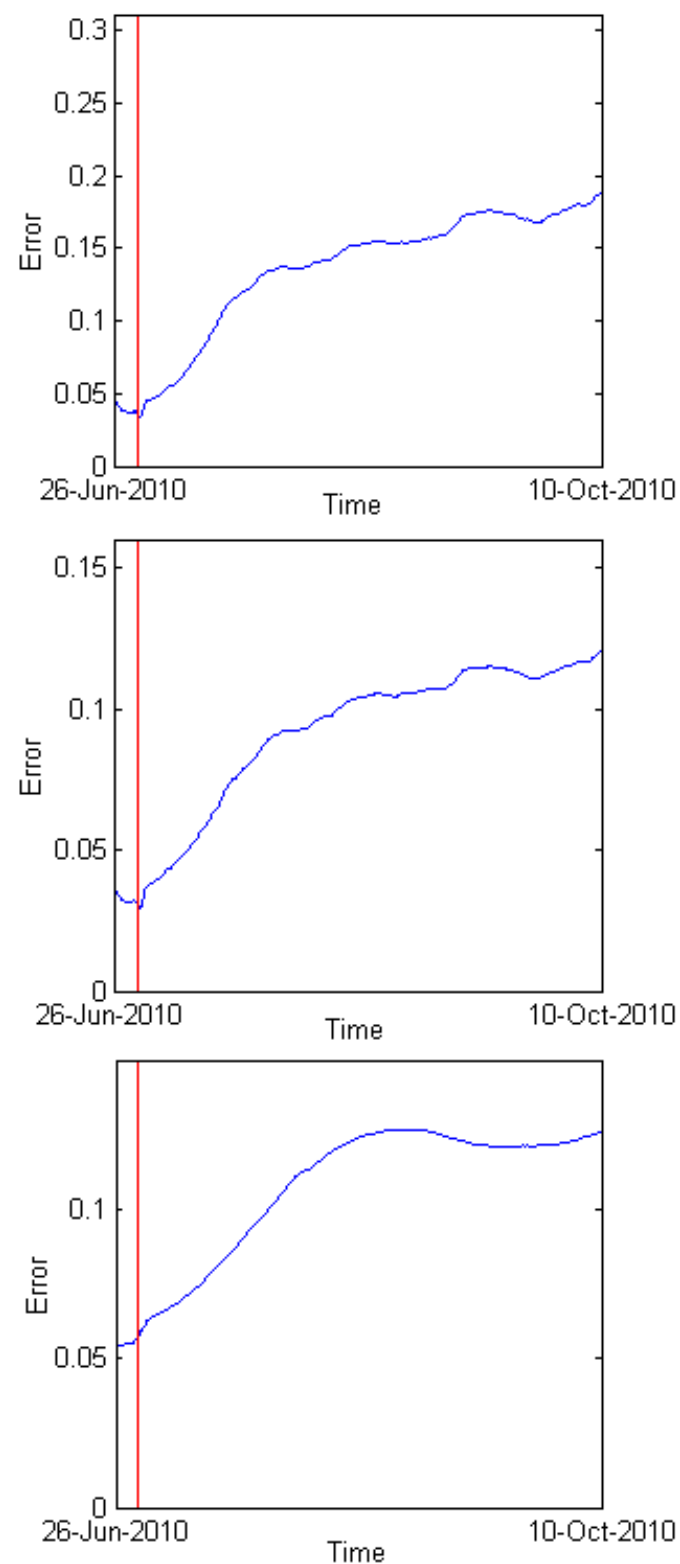

Figure 6: Modulus of the 10000 point moving average error between predicted and actual output for selected sensors and events. Fig. 6A depicts the error for tilt sensors 2 (top), 3 (middle) and 8 (bottom) for the first intervention, while Fig. 6B depicts the error for tilt sensors 2 (top), 3 (middle) and 8 (bottom) for the second intervention. Solid vertical lines indicate damage events. Note the different y axis scales throughout and that in $6 \mathrm{~A}$, the sharp dip shortly after the event marker is due to a zero crossing of the error signal.

By comparing Figs 1, 5 and 6, a picture of both the initial effect of the intervention and the slower long term response emerges. According to the ground truth data, the water tanks were loaded onto the cantilever closer to sensor 1 . The application of this weight seems to have caused the bridge to pivot on the column nearer to the test cantilever, where sensor 7 is located. The impact of the resultant force was most strongly observed during the intervention at sensor 1 . However, the longer term effect was also pronounced between sensors 2 and 3 , as shown by the increase in error from the ESN, but the pivoting also applied a tensile force to the farther column, where tilt sensor 8 is located. The error continued to increase over a period of months as the structural state of the bridge continued to change even after the cessation of this particular intervention. The subsequent levelling out of the ESN error in sensors 2 and 3 suggests that the bridge then reached a state of equilibrium. The fact that this new equilibrium was then sustained suggests that the disposition of the bridge had been permanently changed at this point, with a strained state existing between sensors 2 and 3, even though the column at the far end of the bridge and the cantilever finally returned to their initial states.

The second of the key events came about due to the loading and unloading of two half-full tanks on the bridge between the 
30 th of June 2010 and the 2nd of July 2010. By this time, the region around sensors 2 and 3 had recovered somewhat from the event marked in Fig. 6A. The effects of this second intervention can be seen in sensors 2, 3, and 8, as shown in Fig. $6 \mathrm{~B}$, and are very similar to those produced by the first major event. For tilt sensor 2, the error rose from 0.03 to 0.19 , while for sensor 3 it rose from 0.03 to 0.12 and for sensor 8 from 0.06 to 0.13 . In addition to this, a change in the ESN error signal was seen in the data from every sensor, which suggests that the tests had an impact that could be felt across the whole bridge, although only errors in the areas around sensors 2,3 and 8 were suggestive of permanent damage. It is probable that the loading resulted in the bridge again pivoting on the first column, exacerbating the damage already caused in August 2009. However, in this case the effect on the column was more long lasting.

The way in which the biggest short term effect was seen in the unsmoothed data for tilt sensor 1, but that the biggest long term effect was seen in the smoothed data for sensors 2 and 3 is suggestive of a new optimal approach for structural health monitoring using ESNs:

(i) the unsmoothed data can be used to detect the onset of events;

(ii) the smoothed data can then be used to detect the long term effects of any detected events on the rest of the bridge.

It is interesting to note that some of the events that might have been expected to have caused long term problems do not appear to have had any significant effect. For example, in July 2011, static tests involving the water tanks were performed and some of the steel reinforcing bars in the bridge were cut. Although a major immediate response was seen in the tilt sensors using this technique, no further long-term damage was indicated by the ESN error. The same can be said for the other two events that involved the cutting of reinforcing bars, which occurred in October 2010 and April 2011. Similarly, a creep test in late October 2011, wherein a heavy weight was loaded on the bridge over 17 days, was found to have some effect on the data for sensors 1, 2, 4 and 5 while the event was on-going, but once the test had finished the data suggest that the bridge reverted back to its previous state. Some interventions, such as the first event seen in Fig. 5, were followed almost immediately by another intervention, making it impossible to determine the long term effects of the initial event.

\section{CONCLUSION}

ESNs have been successfully applied in a civil engineering context. It was found that they can predict the output of eight tilt sensors when given an input of ten temperature sensors and this has made it possible to detect both the initial and long term responses of the bridge to a programme of planned manual interventions. All interventions which caused a deviation of the tilt sensors from their predicted behaviour were identified, although medium-term distortion or long-term damage was noted in the tilt sensor data in only a few instances. Two events in particular were found to permanently affect the bridge, one being a test where some water tanks were suspended from one end of the bridge and filled, and another coming almost a year later when heavy water tanks were again suspended from the bridge. In each case, the permanent nature of the damage was identified by noting that the error between the data predicted by the ESNs and the actual sensor data increased both significantly and permanently. By comparing the sensors that produced signals suggesting permanent damage with their location on the bridge, it was possible to make inferences that then allowed the type of damage to be characterised. In the case of the first damage event, it was possible to deduce that the weight applied to the bridge led to its pivoting on one of its columns. This caused changes to the state of the bridge in the region around sensors 2 and 3 over a number of months, before it eventually reached a new equilibrium state. In the case of the second key event, it appeared that the damage from the August 2009 loading of water tanks was exacerbated by repeating the process with half-full tanks, causing permanent damage in a number of regions across the whole bridge.

One final potential avenue for further exploration would be the use of additional ESNs as classifiers. One of the advantages of ESNs is that while they can be used for regression, which was the basis for our work here, they can also be used for classification. A classifying ESN could take the eight real tilt sensors as inputs and be trained to output +1 upon detecting the onset of a significant intervention and -1 during normal behaviour. The performance of these ESN classifiers could be compared to the performance of other established classification techniques, such as Support Vector Machines and Extreme Learning Machines. A system that combined the approach used in this paper with these kinds of classifiers could be of great value to industry.

\section{ACKNOWLEDGMENT}

The authors thank Elena Barton of the UK National Physical Laboratory for providing us with the data from the NPL footbridge project.

\section{REFERENCES}

[1] J. B. Butcher, T. K. Kyriacou, C. R. Day, and P. W. Haycock, "Detection of anomalies present in reinforced concrete bridge data using NARMAX and Echo State Networks," 2012.

[2] V. Livina, E. Barton, and A. Forbes, "Tipping point analysis of the NPL footbridge," Journal of Civil Structural Health Monitoring, vol. 3, pp. 1-8, 2013.

[3] E. Barton and T. Esward, "The Origins of Measurement Uncertainty in SHM - NPL Footbridge Case Study," in 6th European Workshop on Structural Health Monitoring, 2012.

[4] R. Kromanis and P. Kripakaran, "Predicting thermal response of bridges using regression models derived from measurement histories," Computers \& Structures, vol. 136, no. 0, pp. 64-77, 2014.

[5] K. Worden, E. Cross, and E. Barton, "Damage Detection on the NPL Footbridge Under Changing Environmental Conditions," in 6th European Workshop on Structural Health Monitoring, 2012.

[6] H. Jaeger, "Adaptive Nonlinear System Identification with Echo State Networks," in Advances in Neural Information Processing Systems 15, 2002.

[7] M. Lukosevicius and H. Jaeger, "Reservoir computing approach to recurrent neural network training," Computer Science Review, vol. 3, no. 3, pp. 127-149, Aug. 2009.

[8] H. Jaeger and H. Haas, "Harnessing Nonlinearity: Predicting Chaotic Systems and Saving Energy in Wireless Communication," Science, vol. 304, no. 5667, pp. 78-80, 2004.

[9] E. Antonelo, B. Schrauwen, and D. Stroobandt, "Mobile robot control in the road sign problem using Reservoir Computing 
networks," in Robotics and Automation, 2008. ICRA 2008. IEEE International Conference on, 2008, pp. 911-916.

[10] S. Chessa, C. Gallicchio, R. Guzman, and A. Micheli, "Robot Localization by Echo State Networks Using RSS," in Recent Advances of Neural Network Models and Applications, Springer, 2014, pp. 147-154.

[11] M. Oubbati, B. Kord, P. Koprinkova-Hristova, and G. Palm, "Learning of embodied interaction dynamics with recurrent neural networks: some exploratory experiments," Journal of neural engineering, vol. 11, no. 2, p. 026019, 2014.

[12] C. H. Valencia, M. M. B. R. Vellasco, and K. T. Figueiredo, "Trajectory Tracking Control Using Echo State Networks for the CoroBot's Arm," in Robot Intelligence Technology and Applications 2, Springer, 2014, pp. 433-447.

[13] D. Verstraeten, B. Schrauwen, D. Stroobandt, and J. V. Campenhout, "Training word recognition with a liquid state machine: a case study," Information Processing Letters, vol. 95, no. 6, pp. 521-528, 2005.

[14] G. Holzmann, "Reservoir Computing: A powerful black-box framework for nonlinear audio processing," in 12th International Conference on Digital Audio Effects, 2009.

[15] A. Deihimi and H. Showkati, "Application of echo state networks in short-term electric load forecasting," Energy, vol. 39, no. 1, pp. 327340, 2012.

[16] D. P. Hunt, D. Parry, and S. Schliebs, "Exploring the applicability of Reservoir methods for Classifying Punctual Sports Activities Using
On-body Sensors," in Proceedings of the Thirty-Seventh Australasian Computer Science Conference (ACSC 2014), Auckland, New Zealand, 2014.

[17] J. -h. Park, S. -i. Han, and J. -s. Kim, "Improvement of Shape Recognition Performance of Sendzimir Mill Control Systems Using Echo State Neural Networks," Journal of Iron and Steel Research, International, vol. 21, no. 3, pp. 321-327, 2014.

[18] J. Quevedo, H. Chen, M. A. Cuguero, P. Tino, V. Puig, D. Garcia, R. Sarrate, and X. Yao, "Combining learning in model space fault diagnosis with data validation/reconstruction: Application to the Barcelona water network," Engineering Applications of Artificial Intelligence, vol. 30, no. 0, pp. 18-29, 2014.

[19] J. B. Butcher, C. R. Day, P. W. Haycock, D. Verstraeten, and B. Schrauwen, "Defect Detection in Reinforced Concrete using Random Neural Architectures," Computer-Aided Civil and Infrastructure Engineering, vol. 29, no. 3, pp. 191-207, 2014.

[20] D. C. Montgomery, E. A. Peck, and C. G. Vining, Introduction to Linear Regression Analysis. Wiley, 1982.

[21] M. Lukosevicius, "A practical guide to applying echo state networks," in Neural Networks: Tricks of the Trade, 2nd ed., vol. 7700, G. Montavon, G. B. Orr, and K.-R. Muller, Eds. Springer Berlin Heidelberg, 2012, pp. 659-686.

[22] D. Verstraeten, B. Schrauwen, M. D'Haene, and D. Stroobandt, "An experimental unification of reservoir computing methods," Neural Networks, vol. 20, no. 3, pp. 391-403, 2007. 